\title{
Validation and Application of Skin RT-QuIC to Patients in China with Probable CJD
}

\author{
Kang Xiao ${ }^{1,2}$, Xuehua Yang ${ }^{1,2}$, Wei Zhou ${ }^{1,2}$, Cao Chen ${ }^{1,2,3}$, Qi Shi ${ }^{1,2,4, *}$ and Xiaoping Dong ${ }^{1,2,3,4,5, *}$ \\ 1 State Key Laboratory for Infectious Disease Prevention and Control, National Institute for Viral Disease \\ Control and Prevention, Chang-Bai Rd 155, Beijing 102206, China; xiaokang@ivdc.chinacdc.cn (K.X.); \\ yangxuehua@ipbcams.ac.cn (X.Y.); zhouwei@ivdc.chinacdc.cn (W.Z.); chencao@ivdc.chinacdc.cn (C.C.) \\ 2 Collaborative Innovation Center for Diagnosis and Treatment of Infectious Diseases, Zhejiang University, \\ Hangzhou 310007, China \\ 3 Center for Biosafety Mega-Science, Chinese Academy of Sciences, Wuhan 430071, China \\ 4 China Academy of Chinese Medical Sciences, Dongzhimeinei, South Rd 16, Beijing 100700, China \\ 5 Center for Global Public Health, Chinese Center for Disease Control and Prevention, Chang-Bai Rd 155, \\ Beijing 102206, China \\ * $\quad$ Correspondence: shiqi@ivdc.chinacdc.cn (Q.S.); dongxp@ivdc.chinacdc.cn (X.D.); Fax: +86-10-58900815 (X.D.)
}

Citation: Xiao, K.; Yang, X.; Zhou, W. Chen, C.; Shi, Q.; Dong, X. Validation and Application of Skin RT-QuIC to Patients in China with Probable CJD. Pathogens 2021, 10, 1642. https:// doi.org/10.3390/pathogens10121642

Academic Editor: Joaquín Castilla

Received: 6 August 2021

Accepted: 18 November 2021

Published: 19 December 2021

Publisher's Note: MDPI stays neutral with regard to jurisdictional claims in published maps and institutional affiliations.

Copyright: (c) 2021 by the authors. Licensee MDPI, Basel, Switzerland. This article is an open access article distributed under the terms and conditions of the Creative Commons Attribution (CC BY) license (https:// creativecommons.org/licenses/by/ $4.0 /)$.

\begin{abstract}
The definite diagnosis of human sporadic Creutzfeldt-Jakob disease (sCJD) largely depends on postmortem neuropathology and $\mathrm{PrPS}^{\mathrm{Sc}}$ detection in the brain. The development of real-time quaking-induced conversion (RT-QuIC) of cerebrospinal fluid (CSF) samples makes it possible for premortem diagnosis for SCJD. To test the diagnostic potential of RT-QuIC of skin specimens for probable sCJD, we collected the paired skin and CSF samples from 51 recruited living patients referred to the Chinese CJD surveillance center, including 34 probable SCJD, 14 non-CJD, and 3 genetic prion disease ( $\mathrm{gPrD}$ ). The samples were subjected to RT-QuIC assays using recombinant hamster PrP protein $\mathrm{rHaPrP} 90-231$ as the substrate. Using skin RT-QuIC assay, 91.2\% (31/34) probable sCJD patients, and 1 T188K genetic CJD (gCJD) cases showed positive prion-seeding activity, while 85.7\% $(12 / 14)$ non-CJD patients were negative. CSF RT-QuIC positive seeding activity was only observed in 14 probable sCJD patients. Analysis of the reactivity of 38 positive skin RT-QuIC tests revealed that the positive rates in the preparations of $10^{-2}, 10^{-3}$ and $10^{-4}$ diluted skin samples were $88.6 \%$ (39/44), 63.6\% (28/44), and 25.0\% (11/44), respectively. Eleven probable sCJD patients donated two skin specimens collected at different sites simultaneously. Although 95.5\% (21/22) skin RT-QuIC elicited positive reaction, the reactivity varied. Our preliminary data indicate high sensitivity and specificity of skin RT-QuIC in prion detection for Chinese probable sCJD and highlight that skin prion-seeding activity is a reliable biomarker for premortem diagnosis of human prion disease.
\end{abstract}

Keywords: prion disease; sCJD; RT-QuIC; skin; diagnosis; biomarker; seeding activity

\section{Introduction}

Human prion disease $(\operatorname{PrD})$ is a group of transmissible neurodegenerative diseases, consisting of sporadic form, e.g., sporadic Creutzfeldt-Jakob disease (sCJD), genetic or familial form, e.g., genetic CJD (gCJD), fatal familial insomnia (FFI), Gerstmann-SträusslerScheinker disease (GSS), and acquired form, e.g., iatrogenic CJD (iCJD) and variant CJD (vCJD) [1-3]. sCJD is the most common form of human PrD with the incidence of one to two patients per million per year. The definite diagnosis of human PrD, particularly for sCJD, is still largely dependent on postmortem brain tissues showing special neuropathological changes, i.e., spongiform degeneration, and/or $\mathrm{PrPSc}^{\mathrm{Sc}}$ deposit [2], although some biomarkers in cerebrospinal fluid (CSF) have revealed significances in diagnosis of sCJD, such as 14-3-3 and tau [4].

In the past decade, the diagnostic predicament for CJD has been greatly improved due to the development of the real-time quaking-induced conversion (RT-QuIC) assay [5]. The 
implementation of RT-QuIC in the detection of prions in CSF samples has been verified to be of the meaningful diagnostic value for SCJD clinically $[5,6]$. Some countries, such as the USA and UK, have even listed the CSF RT-QuIC test in the national diagnostic standard or criteria for SCJD. However, the sensitivities of CSF RT-QuIC assays in SCJD diagnosis may vary among the different laboratories, ranging from $60 \%$ to almost $100 \%$ [5,7-10]. On the other hand, collecting CSF samples through spinal taps is an invasive process in clinics, and some patients fail to provide CSF due to contraindications or refusal of lumbar puncture. The feasibility of usage of RT-QuIC in other easily obtained specimens is mostly desired.

Recently, the detection of prions in skin specimens with a RT-QuIC test has been reported by Zou's team. They have found that prions could be detected from the autopsy skin tissues of sCJD and vCJD patients [11]. Based on animal experiments, they have also proved that prions could be detected in skin tissues even before the onset of clinical symptoms [12]. These results highlight a good applied prospect of skin RT-QuIC in the premortem diagnosis of human PrDs. In this study, we obtained the paired skin and CSF samples from 51 recruited living patients referred to the Chinese CJD surveillance center, including 34 probable sCJD, 3 gPrD and 14 non-CJD. Positive reaction in a skin RT-QuIC assay was observed in 34 sCJD cases. Meanwhile, only 14 out of 34 probable sCJD were positive in CSF RT-QuIC tests. Our results indicated that a skin specimen was ideal for a RT-QuIC test in Chinese patients.

\section{Results}

Our CSF RT-QuIC assay was blindly evaluated previously with a sensitivity of 96.7\% and a specificity of $100 \%$ by CSF samples of neuropathologically confirmed 30 definite sCJD and 30 non-CJD provided kindly by the NPDPSC [13]. In this study, the skin samples of the coded 10 definite SCJD patients and 5 non-CJD controls from NPDPSC with a dilution from $10^{-2}$ to $10^{-4}$ were examined blindly by our RT-QuIC assay. All the samples were from the site of the lower back. The main demographic information of these 15 subjects is summarized in Table 1. RT-QuIC assays revealed the positive reactions in all definite sCJD samples and negative reactions in all non-CJD samples (Table 1), indicating 100\% sensitivity and specificity of our RT-QuIC assay for prion detection in the skin samples of sCJD and controls.

Table 1. The demographic information of 15 cases from the US Creutzfeldt-Jakob disease (CJD) surveillance center.

\begin{tabular}{|c|c|c|c|c|}
\hline Case No. & Onset Age & Gender $^{1}$ & Diagnosis & Skin RT-QuIC \\
\hline Case 1 & 68 & $\mathrm{~F}$ & Non-CJD & - \\
\hline Case 2 & 78 & $\mathrm{M}$ & Non-CJD & - \\
\hline Case 3 & 84 & $\mathrm{~F}$ & Non-CJD & - \\
\hline Case 4 & 65 & M & Non-CJD & - \\
\hline Case 5 & 58 & M & Non-CJD & - \\
\hline Case 6 & 68 & M & $\mathrm{sCJD}^{2}{\mathrm{MV} 1-2^{3}}^{3}$ & + \\
\hline Case 7 & 59 & $\mathrm{~F}$ & sCJD MM1 & + \\
\hline Case 8 & 65 & $\mathrm{~F}$ & sCJD MM1-2 & + \\
\hline Case 9 & 67 & M & sCJD MM1 & + \\
\hline Case 10 & 79 & M & sCJD MV2 & + \\
\hline Case 11 & 60 & $\mathrm{~F}$ & sCJD MV1-2 & + \\
\hline Case 12 & 57 & $\mathrm{~F}$ & sCJD MM1 & + \\
\hline Case 13 & 71 & $\mathrm{~F}$ & sCJD VV2 & + \\
\hline Case 14 & 78 & $\mathrm{~F}$ & sCJD MM1 & + \\
\hline Case 15 & 68 & $\mathrm{~F}$ & sCJD MM1 & + \\
\hline
\end{tabular}

${ }^{1}$ Female (F); Male (M); ${ }^{2}$ sporadic Creutzfeldt-Jakob disease; ${ }^{3}$ methionine (M); valine (V). 
The paired skin and CSF samples from 51 patients with different clinical diagnoses were enrolled for RT-QuIC assays in this study. The main demographic, clinical examinations and laboratory tests of the 51 patients were summarized in Table 2. The median of the onset age of 34 probable sCJD cases was 61.3 years (y) old (ranging from 28 to $84 \mathrm{y}$ ), while that of 14 non-CJD cases was 62.8 y (43-83 y). The majority of the patients were MM homozygous at codon 129 and EE homozygous at codon 219 of the prion protein gene (PRNP). Periodic sharp wave complexes (PSWC) on EEG were recorded in 8 of $31 \mathrm{sCJD}$ cases and the T188K gCJD case, but not in 13 non-CJD cases who undertook the examination of EEG. sCJD associated abnormalities (high signal in caudate/putamen and/or symmetrical or dissymmetrical cortical ribbon syndrome on diffusion-weighted imaging (DWI)) were noticed in 24 of 33 sCJD patients, T188K and G114V gCJD cases, as well as 8 of 12 non-CJD cases, particularly the cortical ribbon syndrome. CSF 14-3-3 protein was positive in 16 of $30 \mathrm{sCJD}$ cases, the G114V gCJD case, but also in 5 of 14 non-CJD patients (Table 2).

Table 2. The main clinical and laboratory information of the patients with different diseases from Chinese CJD surveillance system.

\begin{tabular}{|c|c|c|c|c|c|c|c|c|c|c|}
\hline Diagnosis & $\begin{array}{l}\text { Onset } \\
\text { Age }\end{array}$ & Gender & $\begin{array}{l}\text { Codon } \\
129^{3}\end{array}$ & $\begin{array}{l}\text { Codon } \\
219^{4}\end{array}$ & EEG $^{5}$ & MRI & $\begin{array}{l}\text { CSF } \\
14-3-3\end{array}$ & $\begin{array}{c}\text { CSF } \\
\text { RT-QuIC }\end{array}$ & $\begin{array}{c}\text { Skin } \\
\text { RT-QuIC }^{1}\end{array}$ & Skin Sampling Site \\
\hline \multicolumn{11}{|l|}{$\begin{array}{l}\text { Probable } \\
\text { sCJD }^{2}\end{array}$} \\
\hline Case 1 & 64 & $\mathrm{~F}$ & MM & $\mathrm{EE}$ & + & - & NA & NA & + & medial upper arm \\
\hline Case 2 & 63 & $\mathrm{~F}$ & MM & $\mathrm{EE}$ & - & + & - & - & + & chest \\
\hline Case 3 & 26 & $\mathrm{~F}$ & MM & $\mathrm{EE}$ & - & + & - & - & + & medial upper arm \\
\hline Case 4 & 64 & $\mathrm{M}$ & MM & $\mathrm{EE}$ & NA & + & - & + & + & medial upper arm \\
\hline Case 5 & 44 & $\mathrm{~F}$ & MM & $\mathrm{EE}$ & - & + & + & - & + & abdomen \\
\hline Case 6 & 59 & $\mathrm{~F}$ & MM & $\mathrm{EE}$ & - & + & - & + & + & medial upper arm \\
\hline Case 7 & 72 & $\mathrm{~F}$ & MM & $\mathrm{EE}$ & - & + & - & - & + & abdomen \\
\hline Case 8 & 70 & $\mathrm{M}$ & MM & EE & - & - & + & - & + & abdomen \\
\hline Case 9 & 69 & $\mathrm{~F}$ & $\mathrm{NA}^{6}$ & NA & + & + & + & - & + & inner thigh \\
\hline Case 10 & 64 & $\mathrm{~F}$ & MM & $\mathrm{EE}$ & - & NA & + & + & + & medial upper arm \\
\hline Case 11 & 68 & $\mathrm{M}$ & MM & $\mathrm{EE}$ & + & + & - & + & + & posterior neck \\
\hline Case 12 & 66 & M & MM & $\mathrm{EE}$ & - & + & - & + & + & medial upper arm \\
\hline Case 13 & 62 & $\mathrm{M}$ & MV & $\mathrm{EE}$ & - & - & + & + & + & medial upper arm \\
\hline Case 14 & 66 & $\mathrm{~F}$ & MM & $\mathrm{EE}$ & + & - & + & - & + & inner thigh \\
\hline Case 15 & 61 & M & MM & $\mathrm{EE}$ & + & + & NA & NA & + & medial upper arm \\
\hline Case 16 & 70 & M & MM & $\mathrm{EE}$ & NA & + & + & + & + & medial upper arm \\
\hline Case 17 & 54 & M & MM & $\mathrm{EE}$ & - & + & + & + & + & medial upper arm \\
\hline Case 18 & 57 & M & MM & $\mathrm{EE}$ & NA & + & + & - & + & medial upper arm \\
\hline Case 19 & 63 & $\mathrm{M}$ & MM & $\mathrm{EE}$ & - & - & + & - & + & medial upper arm \\
\hline Case 20 & 47 & $\mathrm{~F}$ & MM & $\mathrm{EE}$ & - & + & - & + & + & medial upper arm \\
\hline Case 21 & 63 & $\mathrm{M}$ & NA & NA & - & + & - & + & + & abdomen \\
\hline Case 22 & 49 & $\mathrm{~F}$ & MM & EE & - & + & - & - & + & medial upper arm \\
\hline Case 23 & 54 & $\mathrm{~F}$ & MM & $\mathrm{EE}$ & + & + & + & - & + & medial upper arm \\
\hline Case 24 & 69 & $\mathrm{~F}$ & MM & $\mathrm{EE}$ & - & + & - & - & + & posterior neck \\
\hline Case 25 & 55 & $\mathrm{~F}$ & MM & $\mathrm{EE}$ & - & + & + & + & $\begin{array}{l}+ \\
+ \\
+\end{array}$ & $\begin{array}{l}\text { lateral malleolus } \\
\text { inner thigh }\end{array}$ \\
\hline Case 26 & 84 & M & MM & $\mathrm{EE}$ & - & + & + & - & $\begin{array}{l}+ \\
+ \\
+\end{array}$ & $\begin{array}{l}\text { behind ear } \\
\text { medial upper arm }\end{array}$ \\
\hline Case 27 & 52 & M & MM & EE & - & - & + & - & $\begin{array}{l}+ \\
-\end{array}$ & $\begin{array}{l}\text { posterior neck } \\
\text { posterior neck }\end{array}$ \\
\hline Case 28 & 68 & $\mathrm{~F}$ & MM & $\mathrm{EE}$ & + & - & - & + & $\begin{array}{l}- \\
+\end{array}$ & $\begin{array}{l}\text { lateral malleolus } \\
\text { posterior neck }\end{array}$ \\
\hline Case 20 & 00 & $\Gamma$ & IVIVI & $L E$ & $T$ & - & - & $T$ & + & medial upper arm \\
\hline Case 29 & 60 & $\mathrm{~F}$ & MM & EE & + & + & + & - & $\begin{array}{l}- \\
-\end{array}$ & $\begin{array}{l}\text { behind ear } \\
\text { inner thigh }\end{array}$ \\
\hline Case 30 & 67 & $\mathrm{~F}$ & MM & EE & - & - & + & - & + & medial upper arm \\
\hline Case 31 & 53 & M & MM & EE & - & + & NA & NA & $\begin{array}{l}+ \\
- \\
-\end{array}$ & $\begin{array}{c}\text { abdomen } \\
\text { posterior neck }\end{array}$ \\
\hline Case 32 & 81 & $\mathrm{~F}$ & MM & EE & - & - & + & - & + & $\begin{array}{l}\text { Iateral malleorus } \\
\text { inner thigh left } \\
\text { inner thigh right }\end{array}$ \\
\hline Case 33 & 59 & $\mathrm{~F}$ & MM & EE & - & + & + & + & $\begin{array}{l}+ \\
+\end{array}$ & $\begin{array}{l}\text { inner thigh right } \\
\text { lateral malleolus }\end{array}$ \\
\hline Case 34 & 62 & $\mathrm{~F}$ & MM & $\mathrm{EE}$ & - & + & - & + & $\begin{array}{l}+ \\
+ \\
+\end{array}$ & $\begin{array}{l}\text { posterior neck } \\
\text { posterior neck }\end{array}$ \\
\hline Genetic PrD & & & & & & & & & + & laterai malleorus \\
\hline T188K gCJD & 60 & $\mathrm{~F}$ & MM & $\mathrm{EE}$ & + & + & - & + & + & behind ear \\
\hline
\end{tabular}


Table 2. Cont.

\begin{tabular}{|c|c|c|c|c|c|c|c|c|c|c|}
\hline Diagnosis & $\begin{array}{c}\text { Onset } \\
\text { Age }\end{array}$ & Gender & $\begin{array}{l}\text { Codon } \\
129^{3}\end{array}$ & $\begin{array}{l}\text { Codon } \\
219^{4}\end{array}$ & EEG $^{5}$ & MRI & $\begin{array}{c}\text { CSF } \\
14-3-3\end{array}$ & $\begin{array}{c}\text { CSF } \\
\text { RT-QuIC }\end{array}$ & $\begin{array}{c}\text { Skin } \\
\text { RT-QuIC }^{1}\end{array}$ & Skin Sampling Site \\
\hline G114V gCID & 38 & $\mathrm{M}$ & MV & $\mathrm{EE}$ & - & + & + & - & - & behind ear \\
\hline D178N FFI & 66 & $\mathrm{~F}$ & MM & $\mathrm{EE}$ & - & - & - & - & - & medial upper arm \\
\hline Case 1 & 43 & $\mathrm{~F}$ & $\mathrm{MM}$ & $\mathrm{EE}$ & - & + & - & - & - & abdomen \\
\hline Case 2 & 52 & $\mathrm{M}$ & MM & $\mathrm{EE}$ & - & + & - & - & - & behind ear \\
\hline Case 3 & 65 & $\mathrm{M}$ & MM & $\mathrm{EE}$ & - & - & - & - & - & behind ear \\
\hline Case 4 & 68 & $\mathrm{M}$ & MM & EK & NA & NA & - & - & - & medial upper arm \\
\hline Case 5 & 65 & $\mathrm{M}$ & MM & EK & - & - & - & - & - & behind ear \\
\hline Case 6 & 53 & $\mathrm{~F}$ & MM & $\mathrm{EE}$ & - & - & + & - & - & medial upper arm \\
\hline Case 7 & 83 & $\mathrm{~F}$ & NA & NA & - & NA & + & - & - & medial upper arm \\
\hline Case 8 & 57 & $\mathrm{~F}$ & MM & $\mathrm{EE}$ & - & + & + & - & - & inner thigh \\
\hline Case 9 & 69 & $\mathrm{M}$ & MM & $\mathrm{EE}$ & - & + & - & - & - & medial upper arm \\
\hline Case 10 & 69 & $\mathrm{M}$ & $\mathrm{MM}$ & $\mathrm{EE}$ & - & + & + & - & - & abdomen \\
\hline Case 11 & 64 & $\mathrm{M}$ & MM & $\mathrm{EE}$ & - & - & + & - & - & medial upper arm \\
\hline Case 12 & 56 & M & MM & $\mathrm{EE}$ & - & + & - & - & $\begin{array}{l}+ \\
+\end{array}$ & $\begin{array}{l}\text { posterior neck } \\
\text { lateral malleolus }\end{array}$ \\
\hline Case 13 & 80 & $\mathrm{~F}$ & MM & $\mathrm{EE}$ & - & + & - & - & $\begin{array}{l}+ \\
-\end{array}$ & $\begin{array}{c}\text { medial upper arm } \\
\text { abdomen }\end{array}$ \\
\hline Case 14 & 55 & F & MM & $\mathrm{EE}$ & - & + & - & - & $\begin{array}{l}+ \\
-\end{array}$ & $\begin{array}{l}\text { medial upper arm } \\
\text { left } \\
\text { medial upper arm } \\
\text { right }\end{array}$ \\
\hline
\end{tabular}

${ }^{1}$ The results of skin RT-QuIC was based on the final results combined with different dilutions. ${ }^{2}$ sporadic Creutzfeldt-Jakob disease. ${ }^{3}$ Methionine (M); Valine (V). ${ }^{4}$ Glutamicacid (E); Lysine (K). ${ }^{5}$ Positive (+); Negative (-). ${ }^{6}$ Not Available.

Aliquot of $15 \mu \mathrm{L}$ CSF samples from each patient were subjected to RT-QuIC tests. Only $14 \mathrm{sCJD}$ cases and the T188K gCJD case revealed positive reactions, while the rest of the CSF samples tested were negative (Table 2, Figure 1). Differently diluted skin homogenates from 51 patients were also employed in RT-QuIC assays. As shown in Table 2 and Figure 1, most of the sCJD cases were positive (31/34), whereas 12 of 14 non-CJD cases were negative. Additionally, the T188K gCJD cases were also positive in RT-QuIC tests, while the G114V gCJD and D178N FFI cases were negative. Those data highlight that RT-QuIC reactivity or sensitivity of skin samples of SCJD patients is higher than that of CSF samples.

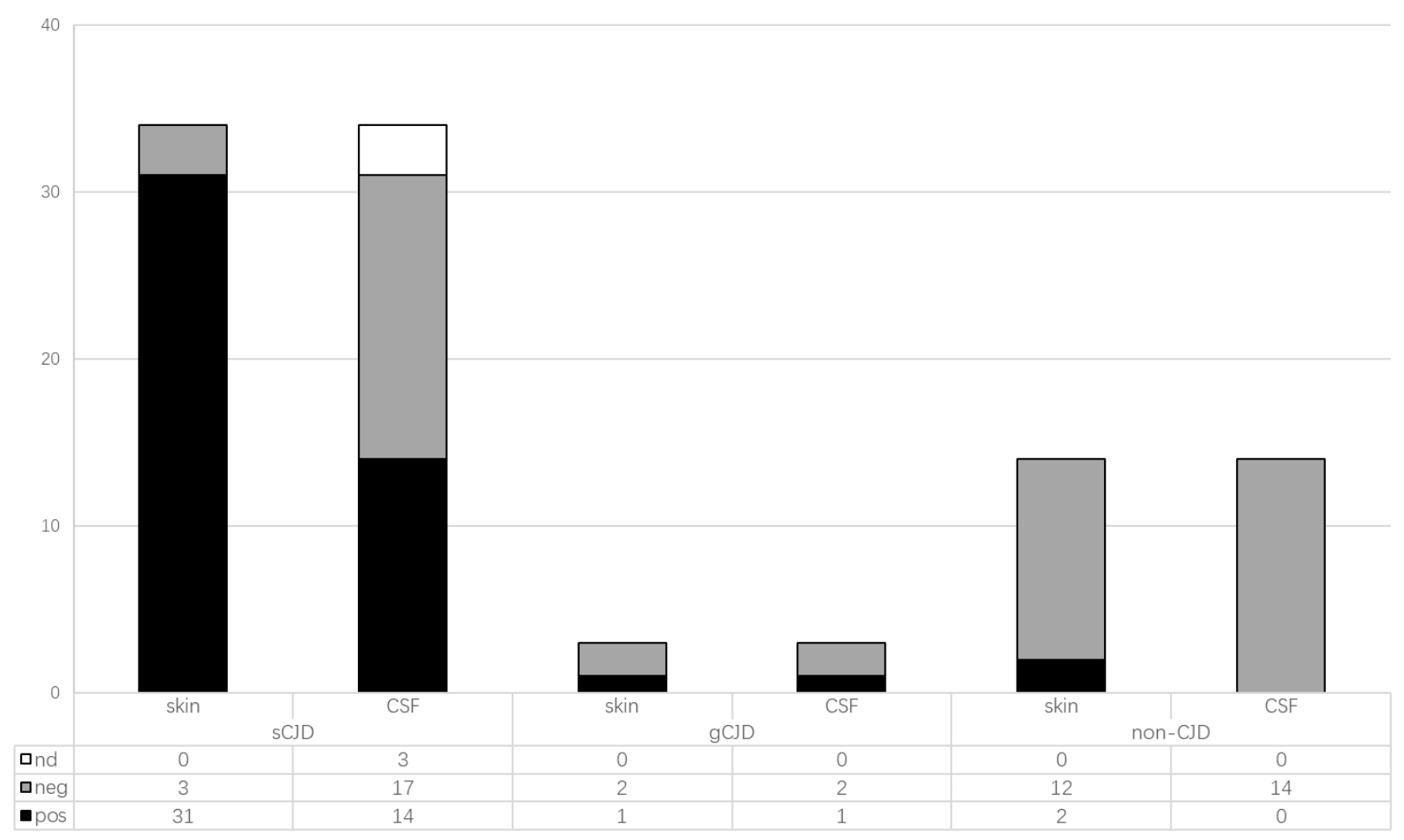

Figure 1. The positive and negative numbers in the skin and CSF RT-QuIC assays among the patients with different diagnosis. 
Furthermore, the RT-QuIC reactivity of each positive skin sample was analyzed based on the dilution of the skin homogenate and the reactivity in every testing well. As shown in Table 3, the general positive rates of the tested skin samples revealed a notable dosedependent pattern, showing $88.6 \%(39 / 44)$ positive rate in the reaction of $10^{-2}$ dilution, $63.6 \%(28 / 44)$ in that of $10^{-3}$ and $25.0 \%(11 / 44)$ in that of $10^{-4}$ dilution. The RT-QuIC positive reactivity of the most tested samples reduced along with the increase of dilution, with four exceptions. Two showed positive reaction in the preparation of $10^{-3}$, but negative in those of $10^{-2}$ and $10^{-4}$, while the other two were positive in the reaction of $10^{-4}$, but negative in that of $10^{-2}$ and $10^{-3}$. It seems that the skin samples of human prion diseases possess the similar dose-dependent RT-QuIC reactive profile as the brain samples.

Table 3. The RT-QuIC reactivity per well of the skin samples at the different dilutions from the patients with various diseases.

\begin{tabular}{|c|c|c|c|c|c|}
\hline \multirow{2}{*}{ Diagnosis } & \multirow{2}{*}{ Skin Sampling Site } & \multicolumn{3}{|c|}{ Dilution $^{2}$} & \multirow{2}{*}{ Final Result } \\
\hline & & $10^{-2}$ & $10^{-3}$ & $10^{-4}$ & \\
\hline \multicolumn{6}{|l|}{ Probable sCJD ${ }^{1}$} \\
\hline Case 1 & medial upper arm & $+/+/+/+$ & $-1-1-1-$ & $-1-1-1-$ & + \\
\hline Case 2 & chest & $+/+/+/+$ & $-1-1-1-$ & $-1-1-1-$ & + \\
\hline Case 3 & medial upper arm & $+/+1+1+$ & $+1+1-1-1$ & $-1-1-1-$ & + \\
\hline Case 4 & medial upper arm & $+/+/+/-$ & $-1-1-1-$ & $-1-1-1-$ & + \\
\hline Case 5 & abdomen & $+/+/+/+$ & $+/+/+/+$ & $-1-1-1-$ & + \\
\hline Case 6 & medial upper arm & $+1+1-1-$ & $+1-1-1-1$ & $+/+/+/+$ & + \\
\hline Case 7 & abdomen & $-1-1+$ & $-/+/+$ & $-1-1+$ & + \\
\hline Case 8 & abdomen & $+/+/+$ & $-1+1+$ & $-1-1-$ & + \\
\hline Case 9 & inner thigh & $-1-1-$ & $-1-1-$ & $+/+/+$ & + \\
\hline Case 10 & medial upper arm & $+/+/+$ & $-1-1-$ & $-1-1-$ & + \\
\hline Case 11 & posterior neck & $+/+/+$ & $+1-1-$ & $-1-1-$ & + \\
\hline Case 12 & medial upper arm & $+/+/+$ & $+1-1-$ & $-1-1-$ & + \\
\hline Case 13 & medial upper arm & $+/+/+/+$ & $+/+/+/+$ & $-1-1-1-$ & + \\
\hline Case 14 & inner thigh & $+/+/+/+$ & $+/+/+/+$ & $+1-1-1-$ & + \\
\hline Case 15 & medial upper arm & $+/+1+/+$ & $+1+1-1-1$ & $-1-1-1-$ & + \\
\hline Case 16 & medial upper arm & $+/+/+/+$ & $+/+1+1-$ & $+1-1-1-$ & + \\
\hline Case 17 & medial upper arm & $+/+/+1+$ & $+1+1-1-1$ & $-1-1-1-$ & + \\
\hline Case 18 & medial upper arm & $+/+/+/+$ & $+/+/+/+$ & $+/+/+/-$ & + \\
\hline Case 19 & medial upper arm & $+/+/+/+$ & $+1+1+1-$ & $+1+1-1-1$ & + \\
\hline Case 20 & medial upper arm & $+/+/+/+$ & $+/+/+/+$ & $+/+/+/+$ & + \\
\hline Case 21 & abdomen & $+/+/+/+$ & $+/+/+/+$ & $+/+/+/+$ & + \\
\hline Case 22 & medial upper arm & $+/+/+1-$ & $-1-1-1-$ & $-1-1-1-$ & + \\
\hline Case 23 & medial upper arm & $+/+/+/+$ & $+/+/+/+$ & $+/+/+/-$ & + \\
\hline \multirow[t]{2}{*}{ Case 24} & posterior neck & $+/+/+/+$ & $+/+/+/-$ & $+/+/+/-$ & + \\
\hline & lateral malleolus & $+/+/+/+$ & $-1-1-1-$ & $-1-1-1-$ & + \\
\hline \multirow[t]{2}{*}{ Case 25} & inner thigh & $+/+/+/+$ & $+1+1-1-1$ & $-1-1-1-$ & + \\
\hline & behind ear & $+/+/+/-$ & $+1-1-1-1$ & $-1-1-1-$ & + \\
\hline \multirow[t]{2}{*}{ Case 26} & medial upper arm & $+/+/+/+$ & $+/+1+1-$ & $-1-1-1-$ & + \\
\hline & posterior neck & $+/+/+/+$ & $+1+1-1-1$ & $+1-1-1-1$ & + \\
\hline \multirow[t]{2}{*}{ Case 28} & posterior neck & $+/+/+/+$ & $+1-1-1-1$ & $-1-1-1-$ & + \\
\hline & medial upper arm & $+/+/+/-$ & $-1-1-1-$ & $-1-1-1-$ & + \\
\hline \multirow[t]{2}{*}{ Case 30} & medial upper arm & $+/+/+/+$ & $+/+/+/+$ & $+1-1-1-1$ & + \\
\hline & abdomen & $+/+1+1+$ & $+1+1+1-$ & $-1-1-1-$ & + \\
\hline \multirow[t]{2}{*}{ Case 32} & inner thigh left & $+/+1+1+$ & $+/+/+/+$ & $+/+1+1-$ & + \\
\hline & inner thigh right & $+/+/+1+$ & $+/+/+/+$ & $+1+1-1-$ & + \\
\hline \multirow[t]{2}{*}{ Case 33} & lateral malleolus & $+/+/+/+$ & $+1+1-1-1$ & $-1-1-1-$ & + \\
\hline & posterior neck & $+/+/+/+$ & $+/+/+/+$ & $+1+1-1-1$ & + \\
\hline \multirow[t]{2}{*}{ Case 34} & posterior neck & $+1-1-1-1$ & $-1-1-1-$ & $-1-1-1-$ & - \\
\hline & lateral malleolus & $+/+/+/+$ & $+1+1-1-1$ & $+1-1-1-1$ & + \\
\hline \multicolumn{6}{|l|}{$\mathrm{gPrD}$} \\
\hline T188K gCJD & behind ear & $+/+/+/+$ & $+/+/+/+$ & $-1-1-1-$ & + \\
\hline
\end{tabular}


Table 3. Cont.

\begin{tabular}{|c|c|c|c|c|c|}
\hline \multirow{2}{*}{ Diagnosis } & \multirow{2}{*}{ Skin Sampling Site } & \multicolumn{3}{|c|}{ Dilution $^{2}$} & \multirow{2}{*}{ Final Result } \\
\hline & & $10^{-2}$ & $10^{-3}$ & $10^{-4}$ & \\
\hline \multicolumn{6}{|l|}{ Non-CJD } \\
\hline Case 12 & posterior neck & $+1-1-1-1$ & $+/+/+/+$ & $-1-1-1-$ & + \\
\hline & lateral malleolus & $+/+/+/-$ & $-1-1-1-$ & $-1-1-1-$ & + \\
\hline Case 14 & medial upper arm left & $+/+/+1-$ & $+1+1-1-$ & $-1-1-1-$ & + \\
\hline & medial upper arm right & $+/-1-1-$ & $-/-/-1-$ & $-/-/-1-$ & - \\
\hline Total (pos/total, \%) & / 1 & $39 / 44(88.6 \%)$ & $28 / 44(63.6 \%)$ & $11 / 44(25.0 \%)$ & / \\
\hline
\end{tabular}

${ }^{1}$ sporadic Creutzfeldt-Jakob disease. ${ }^{2}$ Positive (+); Negative (-).

Fourteen patients (sCJD Cases 24 to 34, as well as non-CJD Cases 12 to 14) donated two skin samples simultaneously, collected from different sites including posterior neck, lateral malleolus, medial forearm, behind ear and inner thigh. Most of these samples $(12 / 14)$ showed consistent results except sCJD Case 34 and non-CJD Case 14. sCJD Case 34 was positive in the sample from lateral malleolus but negative in the posterior neck. Non-CJD Case 14 was positive in the left medial upper arm but not the right arm. The reactivities of these 14 patients were also slightly different. The samples of posterior neck in the SCJD Case 24 and 33 were positive in the reactions of all dilutions $\left(10^{-2}\right.$ to $\left.10^{-4}\right)$, while the samples of lateral malleolus from the same patients elicited positive RT-QuIC reactions only in the dilutions of $10^{-2}$ and $10^{-3}$ (Table 3). By contrast, sCJD Case 34 was positive in the sample of lateral malleolus but negative in the sample of the posterior neck.

To evaluate the potential influence factors for the reactivity in skin RT-QuIC test, 51 tested patients were grouped based on the reactivities of the skin RT-QuIC (34 positive and 17 negative) and the associations with some main data of clinical history, clinical examinations and laboratory tests were analyzed. As shown in Table 4, despite slight differences between the skin RT-QuIC positive and negative groups, no statistical significance was observed in age of disease onset, gender, and polymorphisms at residues s 129 and 219 of PrP. PSWC on EEG seemed to be a positive, related factor to the positive reaction in skin RT-QuIC, as 8 out of 23 patients with positive in skin RT-QuIC reported PSWC on EEG, while only 1 out of 15 cases who were negative in skin RT-QuIC revealed PSWC on EEG, however, without statistical significance $(p=0.06)$. Special abnormalities on MRI were recorded in 25 out of 33 patients (one case not performed) showing the positive skin RT-QuIC, and also observed in 9 out 15 cases (two cases not performed) in the negative group. CSF 14-3-3 positive was detected in 15 out of 32 cases (two case not performed) in the positive group and 7 out 15 cases in the negative one. All 15 patients with CSF RT-QuIC positive were also skin RT-QuIC positive.

Table 4. Comparisons of clinical and laboratory data between skin RT-QuIC positive and negative groups.

\begin{tabular}{cccc}
\hline & Positive & Negative & $p$-Value $\mathbf{1}^{\mathbf{1}}$ \\
\hline Age (median, min-max $)\left(\mathrm{y}^{2}\right)$ & $61.5(26-84)$ & $60.9(38-83)$ & 0.86 \\
Gender $\left(\mathrm{M} / \mathrm{F}^{3}\right)$ & $13 / 21$ & $10 / 7$ & 0.17 \\
Codon 129 $\left(\mathrm{MM} / \mathrm{MV}^{4}\right)$ & $31 / 1$ & $15 / 1$ & 0.62 \\
Codon 219 $\left(\mathrm{EE} / \mathrm{EK}^{5}\right)$ & $32 / 0$ & $14 / 2$ & 0.16 \\
EEG $\left(+/-{ }^{6}\right)$ & $8 / 23$ & $1 / 15$ & 0.06 \\
MRI $(+/-)$ & $25 / 8$ & $9 / 6$ & 0.28 \\
CSF 14-3-3 $(+/-)$ & $15 / 17$ & $7 / 8$ & 0.99 \\
\hline
\end{tabular}

${ }^{1}$ Levene's test and Student's $t$-test were used. $p \leq 0.05$ was considered to be statistically significant. ${ }^{2}$ years (y)

${ }^{3}$ Female (F); Male (M). ${ }^{4}$ Methionine (M); Valine (V). ${ }^{5}$ Glutamicacid (E); Lysine (K). ${ }^{6}$ Positive (+); Negative $(-)$.

\section{Discussion}

CSF RT-QuIC assay is becoming an ideal method for the premortem diagnosis of SCJD and prion research based on its reliable specificity and high sensitivity $[5,8]$. Besides 
RT-QuIC, another similar technique, endpoint quaking-induced conversion (EP-QuIC), was also adopted in some countries such as Canada. Both assays exploited the ability of $\mathrm{PrP}^{\mathrm{Sc}}$ to induce the conversion of $\mathrm{PrP}^{\mathrm{C}}$ into a misfolded form. However, RT-QuIC has a better specificity and a slightly lower sensitivity [14]. Considering that SCJD is a non-communicable disease with nearly $100 \%$ mortality, specificity should be put in the first place. In this study, we tested the feasibility of the skin RT-QuIC assay in the diagnosis for probable sCJD together with the paired CSF samples. We found that 91.1\% (31/34) of probable sCJD cases were positive in skin RT-QuIC assays using recombinant hamster PrP90-231 as the substrate, whereas 14.3\% (2/14) cases who were considered as non-CJD at the time of sampling were positive. It should be noted that these two cases showing positive reactions in the group of non-CJD were lost to follow up. However, at the times of sampling, the clinical manifestations did not meet the diagnostic criteria. Moreover, only $45.2 \%(14 / 31)$ paired CSF samples from those probable SCJD cases showed positive in CSF RT-QuIC. It highlights higher sensitivity of skin RT-QuIC for prion detection in the Chinese probable sCJD cases than that of CSF RT-QuIC. Although the exact reasons why our CSF RT-QuIC assay reveals a lower sensitivity compared to the previous reports by others remain unclear [10], the following possibilities need to be excluded in the future. First, it could be due to the high prevalence of sCJD with $129 \mathrm{MM}$ that was found in all cases except for one and that has been shown to have a lower sensitivity compared to sCJD with 129VV. Second, it could result from some unknown inhibitors in CSF, which shows a race-related tendency, as under the same RT-QuIC experimental condition a very high positive rate (96.7\%) was detected in the CSF samples from US Caucasian patients. Certainly, we cannot exclude the possibility of relatively low sensitivity of the RT-QuIC in our experimental condition, which is able to detect $\mathrm{PrPS}^{\mathrm{Sc}}$ in CSFs of definite SCJD patients but shows low sensitivity in CSFs from probable sCJD cases.

Another point that needs to be emphasized was that since the specific MM type was not clear in Chinese patients, and there was no MM2 type in the definite samples from NPDPSC, we cannot draw a conclusion for skin RT-QuIC of MM2 samples yet.

Our data here revealed a dose-dependent manner of skin prion-seeding activity by the RT-QuIC assay. The highest positive rate was observed in the reactions of the lowest dilution $\left(10^{-2}\right.$ dilution). On the contrary, our study and many other studies have demonstrated that the brain tissues of SCJD patients can induce a positive reaction in RT-QuIC at very high dilution $\left(10^{-8}\right.$ dilution $)[15,16]$. Obviously, the reactivity of RT-QuIC depends on the amount of tissue $\mathrm{PrP}^{\mathrm{Sc}}$. Usually, it is almost impossible to detect $\operatorname{PrP}^{\mathrm{Sc}}$ in the skin specimen of CJD patients with routine techniques, such as protease- or guanidinetreated Western blot and immunohistochemistry, suggesting very low concentration of $\mathrm{PrP}^{\mathrm{Sc}}$ in skin specimens. It is worth noting that a few skin samples in this study exhibited positive reactions at relatively higher dilutions $\left(10^{-3}\right.$ and $\left.10^{-4}\right)$ but failed to elicit positive reactions at low dilution $\left(10^{-2}\right)$. The exact reason is unknown at present. Our previous CSF RT-QuIC assay of human PrDs also revealed a similar phenomenon that excessive amounts of CSF from some patients had a low or no reactivity of prion-seeding activity. It is possible that some unknown inhibitors in human tissues may affect the RT-QuIC reactivity. Relatively shorter lag times and higher fluorescent intensities were observed in the low dilutions $\left(10^{-2}\right.$ and $\left.10^{-3}\right)$ of the skin samples generally (data not shown), but those features varied largely among the tested skin samples. Nevertheless, based on our preliminary data we suggest that it is better to perform skin RT-QuIC assays within a certain range of dilutions, such as $10^{-2}$ and $10^{-3}$ of tissue homogenate, for the diagnosis of human PrDs.

We have also noticed that the skin samples showing positive reactions in skin RT-QuIC assays were collected from different body regions, including neck, upper arm, inner thigh, lateral malleolus, which implies a wide distribution of prion agents in skin tissues of sCJD patients during diseases. Zou and his colleagues have described that the skin specimens of sCJD patients around the head and ear seem to show the highest sensitivity in RT-QuIC test [11]. However, such a phenomenon is uncertain in the current study possibly due to 
limited tested numbers. We recommend taking multiple parts of the skin samples if it is possible to increase the identifying opportunity of prion agents with RT-QuIC.

The skin samples from three different gPrD cases were also included in this study. Only the case of T188K gCJD was positive in the skin RT-QuIC test. T188K gCJD is the second most frequently observed gCJD type in Chinese people [17]. More importantly, T188K gCJD is obviously predominant among Chinese patients, but rarely described in other countries, even in Japan and Korea [18]. Our previous study has revealed that 52\% $(13 / 25)$ T188K gCJD cases are positive in CSF RT-QuIC [19]. Coincidentally, the T188K gCJD case in this study was also positive in CSF RT-QuIC. D178N FFI is the most frequently detected $\mathrm{gPrD}$ in the Chinese [17]. The clinical phenotype and neurological abnormality of D178N FFI are markedly different from GSS and other gCJDs [20]. Our study has verified a remarkably low positive rate and weak reactivity in CSF RT-QuIC of FFI patients compared with the cases of E200K and T188K gCJD [19]. It is not surprising that the FFI case here was negative in skin RT-QuIC. G114V gCJD is rare subtype among the Chinese and only four cases have been identified in our surveillance system since 2006 [17]. Besides the case presented here, another G114V gCJD Chinese case showed negative in CSF RT-QuIC as well (data not shown). Due to the diversity of human $\mathrm{gPrDs}$, the diagnostic significance of skin RT-QuIC needs further analysis.

Reviewing some clinical features of the tested patients with SCJD did not show any significant correlation with the results of skin RT-QuIC. Some SCJD associated clinical examination and laboratory tests seemed to be statistically correlated with the reactivity in skin RT-QuIC, such as PSWC on EEG, implying its accuracy for the diagnosis of SCJD. Therefore, it is most likely that those skin RT-QuIC associated factors probably reflect the close disease-related phenomenon. Although the skin RT-QuIC assay is still at the starting stage, our preliminary data revealed its high sensitivity in probable SCJD patients and reliable specificity in non-CJD cases, which provides a proof of concept that skin RT-QuIC is a suitable tool for premortem diagnosis of SCJD.

\section{Materials and Methods}

\subsection{Samples}

The paired skin and CSF samples from 51 patients with probable sCJD, gPrD or nonCJD were obtained from the tissue bank in the Center of Chinese CJD Surveillance System, including 34 sCJD, 1 T188K gCJD, 1 G114V gCJD, 1 D178N FFI, and 14 cases who were considered as non-CJD cases at the time of sampling. Among them, 14 patients provided two skin samples collected from different sites (Table 2). The demographic information of the patients, the clinical data, MRI and EEG data, the results of the Western blot for CSF 14-3-3 and PRNP sequencing were collected from the database of the Center of Chinese CJD Surveillance System.

In addition, $10 \%$ skin homogenates of neuropathologically-confirmed 10 sCJD patients and 5 non-CJD controls from the National Prion Disease Pathology Surveillance Center (NPDPSC), Case Western Reserve University School of Medicine, Cleveland, Ohio, USA, were also enrolled in this study for validation of our skin RT-QuIC assay. All five nonCJD subjects were determined by neuropathological assays on postmortem brains, which excluded the possibilities of SCJD or other human prion diseases but did not have a diagnosis for other neurological diseases.

All enrolled CSF samples were obtained by lumbar puncture without visible blood. Routine CSF biochemistry assays of those specimens, including cell count, glucose and total protein were all in the normal ranges. To set up our CSF RT-QuIC assay, CSF samples from neuropathologically-confirmed CJD $(n=30)$ and non-CJD $(n=30)$ provided by NPDPSC were used.

\subsection{Preparation of Homogenates of Skin}

The sites for skin biopsies in this study included the skins behind the ears, inside the arms, inside the thighs, lower back, and/or abdomen. After disinfection with 75\% 
alcohol, the patient received local anesthesia with subcutaneous injection of $1-2 \%$ lidocaine hydrochloride. A small piece of skin with a size of about $0.2 \times 0.3 \mathrm{~cm}^{2}$ was taken with a disposable skin biopsy punch (Acupunch, Acuderm Inc., Fort Lauderdale, FL, USA), according to the manufacturer's instruction. Usually, the biopsy skin specimen covers epidermis, dermis, and adipose tissues. We prepared $2 \%(w / v)$ of skin homogenate in lysis buffer $(900 \mu \mathrm{L}$ of TBS containing $2 \mathrm{mM} \mathrm{CaCl} 2$ and $0.25 \%(w / v)$ collagenase $\mathrm{A})$ according to a previously described protocol $[11,12]$. The homogenates were then stored at $-80{ }^{\circ} \mathrm{C}$ for further used.

\subsection{Real-Time Quaking-Induced Conversion RT-QuIC Assays}

The detail procedure of the RT-QuIC assay was described previously [19]. Briefly, RT-QuIC reaction contained $10 \mu \mathrm{g}$ of $\mathrm{rHaPrP} 90-231,1 \mathrm{X}$ PBS, $170 \mathrm{mM} \mathrm{NaCl}, 1 \mathrm{mM}$ EDTA, $0.01 \mathrm{mM}$ ThT, $0.002 \%$ SDS, together with $15 \mu \mathrm{L}$ CSF samples or $2 \mu \mathrm{L} 10^{-2}$ to $10^{-4}$ diluted skin homogenates in a final volume of $100 \mu \mathrm{L}$. Each sample was assayed in triplicate or quadruplicate. The assay was conducted in a black 96-well, optical-bottomed plate (Nunc, 265301) on a BMG FLUOstar plate reader (BMG LABTECH). The main working conditions were fixed as follow: temperature, $55^{\circ} \mathrm{C}$; vibration speed, $700 \mathrm{rpm}$; vibration/incubation time, 60/60 s; total reaction time, $60 \mathrm{~h}$. ThT fluorescence (excitation wavelength, $450 \mathrm{~nm}$; emission wavelength, $480 \mathrm{~nm}$ ) for each reaction was automatically measured every $45 \mathrm{~min}$ and expressed as relative fluorescence units ( $\mathrm{rfu}$ ). The cutoff value was set as the mean value of the negative controls plus 10 times the standard deviation. A sample was considered as positive when $\geq 2$ wells revealed positive reaction curves. The positive control was $10^{-5}$ dilution of the brain homogenate of the scrapie agent 263K-infected hamster, while the negative control was $10^{-5}$ dilution of the brain homogenate of normal hamster.

\subsection{Ethics Approval}

Usage of the CSF and skin samples and relevant clinical information of the patients with different diseases in the Center of Chinese CJD Surveillance System has been approved and monitored by the Ethics Committee of the National Institute for Viral Disease Control and Prevention, China CDC, under the protocol of 2009ZX10004-101 and by the institutional review boards (IRB) of the University Hospital Cleveland Medical Center Written informed consent was obtained from family members for skin autopsy through the NPDPSC.

\subsection{Statistical Analysis}

Statistical analysis was performed using SPSS 17.0 statistical package (Armonk, NY, USA). Levene's test and Student's $t$-test were used as appropriate. $p$ value less than 0.05 was considered to be statistically significant.

Author Contributions: K.X., X.Y. and W.Z. contributed to material preparations, data collections and testing performances. C.C. participated in data analysis. Q.S. and X.D. designed the study, coordinated work and analyzed the data. K.X., Q.S. and X.D. drafted the manuscript. All authors have read and agreed to the published version of the manuscript.

Funding: This work was supported by Chinese National Natural Science Foundation Grants (81630062), SKLID Development Grant (2019SKLID501, 2019SKLID603, 2019SKLID307).

Institutional Review Board Statement: The study was conducted according to the guidelines of the Declaration of Helsinki, and approved by the Ethics Committee of the National Institute for Viral Disease Control and Prevention, China CDC, under the protocol of 2009ZX10004-101 and by the institutional review boards (IRB) of the University Hospital Cleveland Medical Center Written informed consent was obtained from family members for skin autopsy through the NPDPSC.

Informed Consent Statement: Informed consent was obtained from all subjects involved in the study.

Data Availability Statement: All data is available upon request.

Conflicts of Interest: The authors declare no conflict of interest. 


\section{References}

1. Chen, C.; Dong, X.P. Epidemiological characteristics of human prion diseases. Infect. Dis. Poverty 2016, 5, 47. [CrossRef]

2. $\quad$ Prusiner, S.B. Prions. Proc. Nat. Acad. Sci. USA 1998, 95, 13363-13383. [CrossRef]

3. Prusiner, S.B. The prion diseases. Brain Pathol. 1998, 8, 499-513. [CrossRef] [PubMed]

4. Li, Q.X.; Varghese, S.; Sarros, S.; Stehmann, C.; Doecke, J.D.; Fowler, C.J.; Masters Cl Collins, S.J. CSF Tau supplements 14-3-3 protein detection for sporadic Creutzfeldt-Jakob disease diagnosis while transitioning to next generation diagnostics. J. Clin. Neurosci. 2018, 50, 292-293. [CrossRef] [PubMed]

5. McGuire, L.I.; Peden, A.H.; Orrú, C.D.; Wilham, J.M.; Appleford, N.E.; Mallinson, G.; Andrews, M.; Head, M.W.; Caughey, B.; Green, A.J.; et al. Real time quaking-induced conversion analysis of cerebrospinal fluid in sporadic Creutzfeldt-Jakob disease. Ann. Neurol. 2012, 72, 278-285. [CrossRef] [PubMed]

6. Cramm, M.; Schmitz, M.; Karch, A.; Mitrova, E.; Kuhn, F.; Schroeder, B.; Raeber, A.; Varges, D.; Kim, Y.S.; Zerr, I.; et al. Stability and Reproducibility Underscore Utility of RT-QuIC for Diagnosis of Creutzfeldt-Jakob Disease. Mol. Neurobiol. 2016, 53, 1896-1904. [CrossRef] [PubMed]

7. Satoh, K.; Atarashi, R.; Nishida, N. Real-Time Quaking-Induced Conversion for Diagnosis of Prion Disease. Methods Mol. Biol. 2017, 1658, 305-310. [PubMed]

8. Orru, C.D.; Groveman, B.R.; Hughson, A.G.; Zanusso, G.; Coulthart, M.B.; Caughey, B. Rapid and sensitive RT-QuIC detection of human Creutzfeldt-Jakob disease using cerebrospinal fluid. mBio 2015, 6, e02451-14. [CrossRef] [PubMed]

9. Orrú, C.D.; Bongianni, M.; Tonoli, G.; Ferrari, S.; Hughson, A.G.; Groveman, B.R.; Fiorini, M.; Pocchiari, M.; Monaco, S.; Zanusso, G.; et al. A test for Creutzfeldt-Jakob disease using nasal brushings. N. Engl. J. Med. 2014, 371, 519-529. [CrossRef] [PubMed]

10. Foutz, A.; Appleby, B.S.; Hamlin, C.; Liu, X.; Yang, S.; Cohen, Y.; Chen, W.; Blevins, J.; Wang, H.; Safar, J.G.; et al. Diagnostic and prognostic value of human prion detection in cerebrospinal fluid. Ann. Neurol. 2017, 81, 79-92. [CrossRef] [PubMed]

11. Orrú, C.D.; Yuan, J.; Appleby, B.S.; Li, B.; Li, Y.; Winner, D.; Wang, Z.; Zhan, Y.A.; Joshi, T.; Zou, W.Q.; et al. Prion seeding activity and infectivity in skin samples from patients with sporadic Creutzfeldt-Jakob disease. Sci. Transl. Med. 2017, 9, 417. [CrossRef] [PubMed]

12. Wang, Z.; Manca, M.; Foutz, A.; Camacho, M.V.; Raymond, G.J.; Race, B.; Orru, C.D.; Yuan, J.; Shen, P.; Zou, W.Q.; et al. Early preclinical detection of prions in the skin of prion-infected animals. Nat. Commun. 2019, 10, 247. [CrossRef] [PubMed]

13. Xiao, K.; Yang, X.H.; Zou, W.Q.; Dong, X.P.; Shi, Q. Assessment of the Sensitivity and Specificity of the Established Real-time Quaking-induced Conversion (RT-QuIC) Technique in Chinese CJD Surveillance. Biomed. Environ. Sci. 2020, 33, 620-622. [PubMed]

14. Cheng, K.; Vendramelli, R.; Sloan, A.; Waitt, B.; Podhorodecki, L.; Godal, D.; Knox, J.D. Endpoint Quaking-Induced Conversion: A Sensitive, Specific, and High-Throughput Method for Antemortem Diagnosis of Creutzfeldt-Jacob Disease. J. Clin. Microbiol. 2016, 54, 1751-1754. [CrossRef] [PubMed]

15. Peden, A.H.; McGuire, L.I.; Appleford, N.E.; Mallinson, G.; Wilham, J.M.; Orru, C.D.; Caughey, B.; Ironside, J.W.; Knight, R.S.; Head, M.W.; et al. Sensitive and specific detection of sporadic Creutzfeldt-Jakob disease brain prion protein using real-time quaking-induced conversion. J. Gen. Virol. 2012, 93 Pt 2, 438-449. [CrossRef] [PubMed]

16. Orrù, C.D.; Groveman, B.R.; Hughson, A.G.; Manca, M.; Raymond, L.D.; Raymond, G.J.; Campbell, K.J.; Anson, K.J.; Kraus, A.; Caughey, B.; et al. RT-QuIC Assays for Prion Disease Detection and Diagnostics. Methods Mol. Biol. 2017, 1658, $185-203$.

17. Shi, Q.; Zhou, W.; Chen, C.; Zhang, B.Y.; Xiao, K.; Zhang, X.C.; Shen, X.J.; Li, Q.; Deng, L.Q.; Dong, X.P.; et al. The Features of Genetic Prion Diseases Based on Chinese Surveillance Program. PLoS ONE 2015, 10, e0139552. [CrossRef]

18. Shi, Q.; Zhou, W.; Chen, C.; Xiao, K.; Wang, Y.; Gao, C.; Dong, X.P. Rare genetic Creutzfeldt-Jakob disease with T188K mutation: Analysis of clinical, genetic and laboratory features of 30 Chinese patients. J. Neurol. Neurosurg. Psychiatry 2017, 88, 889-890. [CrossRef] [PubMed]

19. Xiao, K.; Shi, Q.; Zhou, W.; Zhang, B.Y.; Wang, Y.; Chen, C.; Ma, Y.; Gao, C.; Dong, X.P. T188K-Familial Creutzfeldt-Jacob Disease, Predominant Among Chinese, has a Reactive Pattern in CSF RT-QuIC Different from D178N-Fatal Familial Insomnia and E200K-Familial CJD. Neurosci. Bull. 2019, 35, 519-521. [CrossRef] [PubMed]

20. Shi, Q.; Chen, C.; Gao, C.; Tian, C.; Zhou, W.; Zhang, B.Y.; Han, J.; Dong, X.P. Clinical and familial characteristics of ten chinese patients with fatal family insomnia. Biomed. Environ. Sci. 2012, 25, 471-475. [PubMed] 